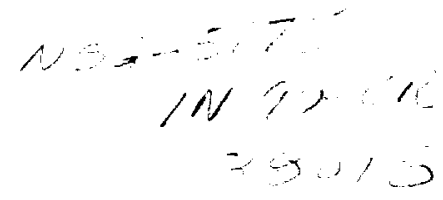

\title{
Phase determination from mostly one-sided interferograms
}

\author{
David G. Johnson, Wesley A. Traub, and Kenneth W. Jucks \\ Smithsonian Astrophysical Observatory, 60 Garden Street, Cambridge, Massachusetts, 02138
}

\begin{abstract}
We show how to detect and correct for non-linear phase shifts in a mainly one-sided interferogram of an emission-line source. We simultaneously detect and correct for an out-of-phase emission background from the spectrometer. The method requires two auxiliary spectra, one of a strong continuum source, and one of an emission-line source with little or no continuum.
\end{abstract}

Key words: Fourier transform spectroscopy, interferometer, phase.

\section{Introduction}

Phase determination for an ideal interferometer amounts to finding the position of zero path difference (ZPD) for each spectral element in a recorded interferogram. In this case, the interferogram $\tilde{F}\left(x^{\prime}\right)$ of a single unresolved spectral line centered at the wavelength $\lambda_{0}=1 / \sigma_{0}$ is given by $\tilde{F}\left(x^{\prime}\right)=a \cos 2 \pi \sigma_{0}\left(x^{\prime}-x\right)$, where $x$ is the position of ZPD and $x^{\prime}$ is the retardation of the retroreflector in the long arm of the interferometer. The instantaneous optical path difference (OPD) is given by $x^{\prime}-x$. In the ideal case where an interferogram sample point falls precisely on the peak of the interferogram we have $x=0$, but in general $x \neq 0$. The transform of a one-sided interferogram gives

$$
\begin{aligned}
F(\sigma) & =\frac{1}{L} \int_{0}^{L} \tilde{F}\left(x^{\prime}\right) e^{i 2 \pi \sigma x^{\prime}} d x^{\prime} \\
& =\frac{a e^{i 2 \pi \sigma_{0} x}}{2}\left[\frac{\sin \alpha}{\alpha}+i \frac{(1-\cos \alpha)}{\alpha}\right],
\end{aligned}
$$


where $L$ is the length of the scan, $\alpha \equiv 2 \pi\left(\sigma-\sigma_{0}\right) L$, and we have dropped terms containing $\left(\sigma+\sigma_{0}\right)$. The transform of a mostly one-sided interferogram (where the integral limits are $-L_{1}$ to $+L_{2}, L_{1} \ll L_{2}$, and a tapering function is applied between $-L_{1}$ and $+L_{1}$ to avoid double-counting of the two-sided part of $\tilde{F}$ ) is similar, but differs in details which are not important here. In either case, once we have determined $x$ the spectrum is recovered by taking the real part of $F(\sigma) e^{-i 2 \pi \sigma_{0} x}$. The transform of a two-sided interferogram (where the mirror scans from $-L$ to $L$ ) for the same unresolved line is simply $a e^{i 2 \pi \sigma_{0} x} \sin \alpha / 2 \alpha$.

The phase of the transform $F(\sigma)$ in Eq. 1 is given by a slowly varying term $\left(2 \pi \sigma_{0} x\right)$, plus a rapidly varying term, $\tan ^{-1}[(1-\cos \alpha) / \sin \alpha]$, which is only significant near a spectral line. We have found that, for our interferometer, the slowly varying term is not well modelled by the linear function $2 \pi \sigma x$. In Sections 3-5 we present a method for detecting and correcting for a small non-linear phase shift. We simultaneously detect and correct for an out-of-phase emission background from the spectrometer.

Eq. 1 illustrates a crucial element of our phase-recovery method, line symmetrizing, which is discussed in Section 4. The phase factor $2 \pi \sigma_{0} x$ in Eq. 1 is not known a priori, but must be estimated. If the estimated phase equals $2 \pi \sigma_{0} x$, then the real part of Eq. 1 gives a symmetric line, from the $\sin \alpha / \alpha$ term. If the estimated phase is $2 \pi \sigma_{0} x \pm \pi / 2$, then the real part of Eq. 1 gives an antisymmetric line, from the $(1-\cos \alpha) / \alpha$ term. In practice, it is straightforward to select the optimum phase factor which symmetrizes the real part of a complex spectral line by multiplying the region around the line by a series of equispaced phase factors, and testing the real part of each result for symmetry.

\section{Instrument description}

The far-infrared spectrometer (FIRS-2) was designed and built at the Smithsonian Astrophysical Observatory for the purpose of measuring stratospheric emission spectra from balloon and aircraft platforms. The spectrometer produces mostly one-sided interferograms, which must be transformed and accurately phase-corrected before the spectra can be used 
to retrieve constituent profiles. The FIRS-2 and data reduction procedure are described in detail elsewhere ${ }^{1,2}$; our phase-recovery method is summarized in the latter ${ }^{2}$, but fully discussed in the present paper. A brief description of the instrument as configured for balloon flights follows.

The flight instrument consists of a limb-scanning telescope, the interferometer, detectors, and associated control electronics. The telescope is a small off-axis reflector which is pointed in elevation relative to a single-axis stabilized platform ${ }^{3,4}$. The interferometer uses hollow corner-cube retroreflectors and a $11 \mu \mathrm{m}$ uncoated Mylar beamsplitter, and scans over optical path differences from $-L_{1}$ to $+L_{2}$, where $L_{1}=1.2 \mathrm{~cm}$ and $L_{2}=120 \mathrm{~cm}$. We use two liquid helium cooled detectors, a gallium-doped germanium photoconductor for the $75-220 \mathrm{~cm}^{-1}$ band and a copper-doped germanium photoconductor for the $330-700 \mathrm{~cm}^{-1}$ band. The field of view is defined by field stops near the detectors.

During a balloon flight we record interferograms for a sequence of elevation angles, repeating the sequence throughout the flight. We first observe an ambient-temperature blackbody source at an elevation angle of $90^{\circ}$, followed by a space view at $30^{\circ}$, and then we observe the limb at elevation angles of $0.0,-2.3,-3.0,-3.6,-4.2$, and $-4.6^{\circ}$. A complete sequence takes about $2700 \mathrm{~s}$. We show small segments of sample flight spectra for 90,30 , and $-4.17^{\circ}$ in Fig. 1, recorded at a balloon altitude of $36 \mathrm{~km}$. The spectra have been phase corrected using the method outlined below, but have not been calibrated in intensity or wavenumber. Note the presence of a substantial out-of-phase (negative) instrumental background in the $30^{\circ}$ spectrum. The phase recovery method discussed here is not affected by this background, which is later detected and corrected for.

\section{Model description}

We assume that the measured blackbody spectrum $M_{90}(\sigma)$ can be modelled as

$$
M_{90}(\sigma)=\left[B(\sigma)+E(\sigma) e^{i \phi(\sigma)}\right] e^{i\left[2 \pi \sigma x_{90}+\epsilon(\sigma)\right]}
$$


where $B(\sigma)$ is the product of the instrumental response function and a blackbody spectrum, $E(\sigma) e^{i \phi(\sigma)}$ is the complex background spectrum ${ }^{5}, x_{90}$ is the position of ZPD in the blackbody interferogram, and $\epsilon(\sigma)$ is the non-linear phase shift.

Similarly, we model the measured space and limb spectra as

$$
\begin{aligned}
& M_{30}(\sigma)=\left[S_{30}(\sigma)+E(\sigma) e^{i \phi(\sigma)}\right] e^{i\left[2 \pi \sigma x_{30}+\epsilon(\sigma)\right]} \\
& M_{\theta}(\sigma)=\left[S_{\theta}(\sigma)+E(\sigma) e^{i \phi(\sigma)}\right] e^{i\left[2 \pi \sigma x_{\theta}+\epsilon(\sigma)\right]}
\end{aligned}
$$

where $S_{30}$ and $S_{\theta}$ are the phase-corrected space and limb spectra, and $x_{30}$ and $x_{\theta}$ are the positions of ZPD in the respective interferograms.

In this notation, the $M_{90,30, \theta}(\sigma)$ functions are complex spectra obtained by applying a fast-Fourier transform to an observed and windowed interferogram, where the window function is typically a weight running linearly from 0 to 1 between $-L_{1}$ and $+L_{1}$, and a constant weight of 1 between $+L_{1}$ and $+L_{2}$. Also in this notation, the functions on the right

sides of Eqns. 2-4 $\left(B(\sigma), S_{30, \theta}(\sigma), E(\sigma), \phi(\sigma), \epsilon(\sigma)\right.$, and $\left.x_{90,30, \theta}\right)$ are all to be extracted from the $M(\sigma)$ data, and therefore include a random noise component. The recovered spectra, $B$ and $S_{30, \theta}$, are phase-corrected, but must be wavenumber ${ }^{2}$ and intensity-calibrated ${ }^{2,5}$ (see also Section 5).

From typical balloon altitudes the space spectrum $S_{30}$ consists of a few narrow emission lines with no continuum emission, the limb spectra $S_{\theta}$ contain many lines superposed on a substantial continuum, and the blackbody spectrum $B$ consists of continuum alone. We assume that the background spectrum $E(\sigma) e^{i \phi(\sigma)}$ is constant for the duration of an observation sequence, and that $\epsilon(\sigma)$ is constant for any given balloon flight. As will be shown in the next section, $\epsilon$ does indeed seem to be constant with respect to time.

\section{Estimate of non-linear phase}

As mentioned in Section 2, each interferogram consists of a low-resolution two-sided part (maximum OPD range of $\pm L_{1}= \pm 1.2 \mathrm{~cm}$ ) and a high-resolution one-sided part (maximum 
OPD of $L_{2}=+120 \mathrm{~cm}$ ). Both the low and high resolution interferograms are used in the steps which follow; we will use $m$ to represent the transform of the low-resolution two-sided interferogram, and $M$ to represent the high-resolution transform. The basic procedure is to estimate $x_{30}$ using the high-resolution spectrum $M_{30}$, estimate $x_{90}$ using the low-resolution spectrum $m_{90}$, and then estimate $\epsilon(\sigma)$ by calculating the phase of the difference $m_{90}-m_{30}$ at low resolution. In the following step-by-step procedure we have omitted some details (tapering functions used for low- and high-resolution transforms, zero-padding, and the like) for brevity and clarity; further details regarding our routine implementation of the fast-Fourier transform are presented in Ref. 2.

Our method differs from one previously described ${ }^{6}$ in that it requires two auxiliary spectra but works for almost totally one-sided interferograms. The radiometric calibration method described in Ref. 5 calibrates and phase-corrects spectra in a single step, but requires saving the complex transforms of hot and cold calibration spectra. Neither method ${ }^{5,6}$ is capable of determining the phase factor $2 \pi \sigma_{0} x$ when the two-sided transform is dominated by the complex background $E(\sigma)$.

Step 1. To begin with, we examine the nominal (theoretical or experimental) spectra to be analyzed, and select a large number (40 to 60 ) of spectral lines which we anticipate should be intrinsically symmetric with respect to reflection about their central wavenumbers. For each line in this group, estimate a value of $x_{30}$ by symmetrizing the line in the full resolution $30^{\circ}$ spectrum. As can be seen by examining Eq. 1, we can determine the phase (modulo $\pi$ ) for a spectral line at $\sigma_{i}$ by finding the phase $\beta_{i}$ for which $M_{30} e^{-i \beta_{i}}$ is symmetric near the line center. We calculate $\bar{x}_{30}$ by fitting the function $\beta(\sigma)=2 \pi \sigma \bar{x}_{30}$ to the derived set of $\left(\sigma_{i}, \beta_{i}\right)$ values. The residuals from the fit are a combination of the error in estimating $\beta_{i}$ and the non-linear phase term $\epsilon\left(\sigma_{i}\right)$. In practice, the uncertainty in $\beta_{i}$ is too large to get a good estimate of $\epsilon\left(\sigma_{i}\right)$ from the residuals.

Step 2. Calculate $m_{30}^{\prime}$, the low-resolution space spectrum with the linear phase removed:

$m_{30}(\sigma) e^{-i 2 \pi \sigma \bar{x}_{30}}=\left[S_{30}+E e^{i \phi}\right] e^{i \epsilon}$, 


$$
\equiv m_{30}^{\prime}
$$

Step 3. Estimate $x_{90}$ by calculating the phase from the transform of the short two-sided part of the blackbody interferogram. We calculate the phase $\beta_{i}$ at each point in regions where $B(\sigma) \gg E(\sigma)$, and again we fit a linear function to the set of $\left(\sigma_{i}, \beta_{i}\right)$ to derive $\bar{x}_{90}$. The residuals from this fit would give a good estimate of $\epsilon(\sigma)$, but only in regions where the background is small.

Step 4. Calculate $m_{90}^{\prime}$, the blackbody spectrum with the linear phase removed:

$$
\begin{aligned}
m_{90}(\sigma) e^{-i 2 \pi \sigma \bar{x}_{90}} & =\left[B+E e^{i \phi}\right] e^{i \epsilon} \\
& \equiv m_{90}^{\prime}
\end{aligned}
$$

Step 5. Calculate the non-linear phase $\epsilon(\sigma)$ :

$$
\begin{aligned}
m_{90}^{\prime}-m_{30}^{\prime} & =\left(B+E e^{i \phi}\right) e^{i \epsilon}-\left(S_{30}+E e^{i \phi}\right) e^{i \epsilon}, \\
& =\left(B-S_{30}\right) e^{i \epsilon} \\
\epsilon(\sigma) & =\tan ^{-1}\left[\frac{\Im\left(m_{90}^{\prime}-m_{30}^{\prime}\right)}{\Re\left(m_{90}^{\prime}-m_{30}^{\prime}\right)}\right] .
\end{aligned}
$$

Step 6. Repeat the process for a number of pairs of $M_{90}$ and $M_{30}$, average the results, and smooth the average to produce the final estimated non-linear phase term $\bar{\epsilon}(\sigma)$.

In Fig. 2 we show $\bar{\epsilon}(\sigma)$ for two sets of measurements made several years apart using different beamsplitters. The estimated phase is unreliable near minima in the beamsplitter efficiency function because in these regions $B \ll E$ and the difference $m_{90}^{\prime}-m_{30}^{\prime}$ is dominated by noise. Outside these regions the two phase estimates are in excellent agreement, indicating that $\epsilon(\sigma)$ is constant in time and independent of beamsplitter thickness. A discussion of the possible physical origin of the non-linear term $\epsilon(\sigma)$ is found in Ref. 5 .

\section{Background estimate and normalization}

Step 7. Estimate the background $E e^{i \phi}$ by clipping lines from the high-resolution phasecorrected spectrum $M_{30}^{\prime} e^{-i \bar{\epsilon}}$ and smoothing the result, as follows. First remove the non-linear phase $\bar{\epsilon}(\sigma)$ from the high-resolution space spectrum $M_{30}^{\prime}$ : 


$$
\begin{aligned}
M_{30}^{\prime} e^{-i \bar{\epsilon}} & =\left[\left(S_{30}+E e^{i \phi}\right) e^{i \epsilon}\right] e^{-i \bar{\epsilon}}, \\
& =S_{30}+E e^{i \phi} .
\end{aligned}
$$

Then effectively subtract $S_{30}$ by clipping the sharp spectral lines from the result:

$$
\begin{aligned}
M_{30}^{\prime} e^{-i \bar{\epsilon}}-S_{30} & =E e^{i \phi}, \\
& \equiv M_{30}^{\prime \prime} .
\end{aligned}
$$

Clipping lines is equivalent to subtracting $S_{30}$ since the continuum emission in the space view is negligible. The clipping is accomplished numerically by replacing each apparent sharp spectral line (above a preset noise threshold) by a straight-line segment which bridges the adjacent spectral regions.

Step 8. Calculate the estimated limb spectrum $S_{\theta}(\sigma)$ for the remaining elevation angles by estimating $\bar{x}_{\theta}$ as for $\bar{x}_{30}$, phase correcting, and subtracting the background as follows:

$$
\begin{aligned}
M_{\theta} e^{-i \bar{\epsilon}} e^{-i 2 \pi \sigma \bar{x}_{\theta}}-M_{30}^{\prime \prime} & =\left(S_{\theta}+E e^{i \phi}\right) e^{i\left(2 \pi \sigma x_{\theta}+\epsilon\right)} e^{-i \bar{\epsilon}} e^{-i 2 \pi \sigma \bar{x}_{\theta}}-E e^{i \phi}, \\
& =S_{\theta}(\sigma) .
\end{aligned}
$$

Step 9. Calculate the observed response $B(\sigma)$ to the blackbody source:

$M_{90}^{\prime} e^{-i \bar{\epsilon}}-M_{30}^{\prime \prime}=B(\sigma)$.

Step 10. Calculate the intensity-calibrated limb spectra $S_{\theta}^{\mathrm{cal}}(\sigma)$ from the derived $S_{\theta}(\sigma)$ and $B(\sigma)$ :

$S_{\theta}^{\text {cal }}(\sigma)=\frac{S_{\theta}(\sigma) P\left(T_{b b}, \sigma\right)}{B(\sigma) P(277 \mathrm{~K}, \sigma)}$

where $P(T, \sigma)$ is the Planck function, $T_{b b}$ is the measured temperature of the reference blackbody source, and $277 \mathrm{~K}$ is a standard reference temperature, chosen for convention in our data analysis procedure. The resulting spectra $S_{\theta}^{\text {cal }}(\sigma)$ are dimensionless and independent of the instrument spectral response, which is convenient for plotting and visualization. Spectra in physical units ( $\mathrm{erg} \mathrm{cm}^{-2} \mathrm{~s}^{-1} \mathrm{st}^{-1}$ ) can easily be recovered by multiplying by $P(277 \mathrm{~K}, \sigma)$. 


\section{Results}

We judge the success or failure of the phase determination by comparing measured and calculated emission line spectra and looking for systematic differences. As mentioned in

Section 4 , the scatter in the estimated phase $\beta_{i}$ is too large to be able to judge the effect of the non-linear phase term on single spectra. In coadded spectra, however, the improvement is considerable in regions where $\epsilon(\sigma)$ is large, particularly in the region $560-610 \mathrm{~cm}^{-1}$ where the non-linear term is as large as $15^{\circ}$. This region is used for fitting $\mathrm{CO}_{2}$ and $\mathrm{N}_{2} \mathrm{O}$ in FIRS2 spectra, and correcting for the non-linear phase term eliminates a systematic difference between observed and calculated line shapes in this region. Spectra which have been phase corrected using the method outlined in this paper have emission line profiles which are visibly more symmetric than results from assuming a linear phase function, as shown in Fig. 3 , and are a much better match to calculated spectra. Failing to correct for the non-linear phase term not only results in a shift in the line position, but also produces highly asymmetric line wings.

\section{Conclusion}

We describe a method for phase correcting and calibrating emission spectra from mostly one-sided interferograms. The method, developed for the FIRS-2, is effective in the presence of an out-of-phase background. The algorithm is in routine use to reduce FIRS-2 spectra, and has been used for several years now with good success.

\section{ACKNOWLEDGMENTS}

We gratefully acknowledge support from NASA's Upper Atmosphere Research Program in the form of grant NSG 5175. 


\section{REFERENCES}

1. W. A. Traub, K. V. Chance, D. G. Johnson, and K. W. Jucks, "Stratospheric spectroscopy with the far-infrared spectrometer (FIRS-2): Overview and recent results," Proc. Soc. Photo-Opt. Instrum. Eng. 1491, 298-307 (1991).

2. D. G. Johnson, K. W. Jucks, W. A. Traub, and K. V. Chance, "Smithsonian stratospheric far-infrared spectrometer and data reduction system," J. Geophys. Res. 100, 3091-3106 (1995).

3. L. M. Coyle, G. Aurilio, G. U. Nystrom, J. Bortz, B. G. Nagy, K. V. Chance, and W. A. Traub, "Design of a single-axis platform for balloon-borne remote sensing," Rev. Sci. Instrum. 57, 2512-2518 (1986).

4. W. A. Traub, K. V. Chance, and L. M. Coyle, "Performance of a single-axis platform for balloon-borne remote sensing," Rev. Sci. Instrum. 57, 2519-2522 (1986).

5. H. E. Revercomb, H. Buijs, H. B. Howell, D. D. LaPorte, W. L. Smith, and L. A. Sromovsky, "Radiometric calibration of IR Fourier transform spectrometers: Solution to a problem with the High-Resolution Interferometer Sounder," Appl. Opt. 27, 3210-3218 (1988).

6. R. C. M. Learner, A. P. Thorne, I. Wynne-Jones, J. W. Brault, and M. C. Abrams, "Phase correction of emission line Fourier transform spectra," J. Opt. Soc. Am. A 12, 2165-2171 (1995). 


\section{FIGURES}

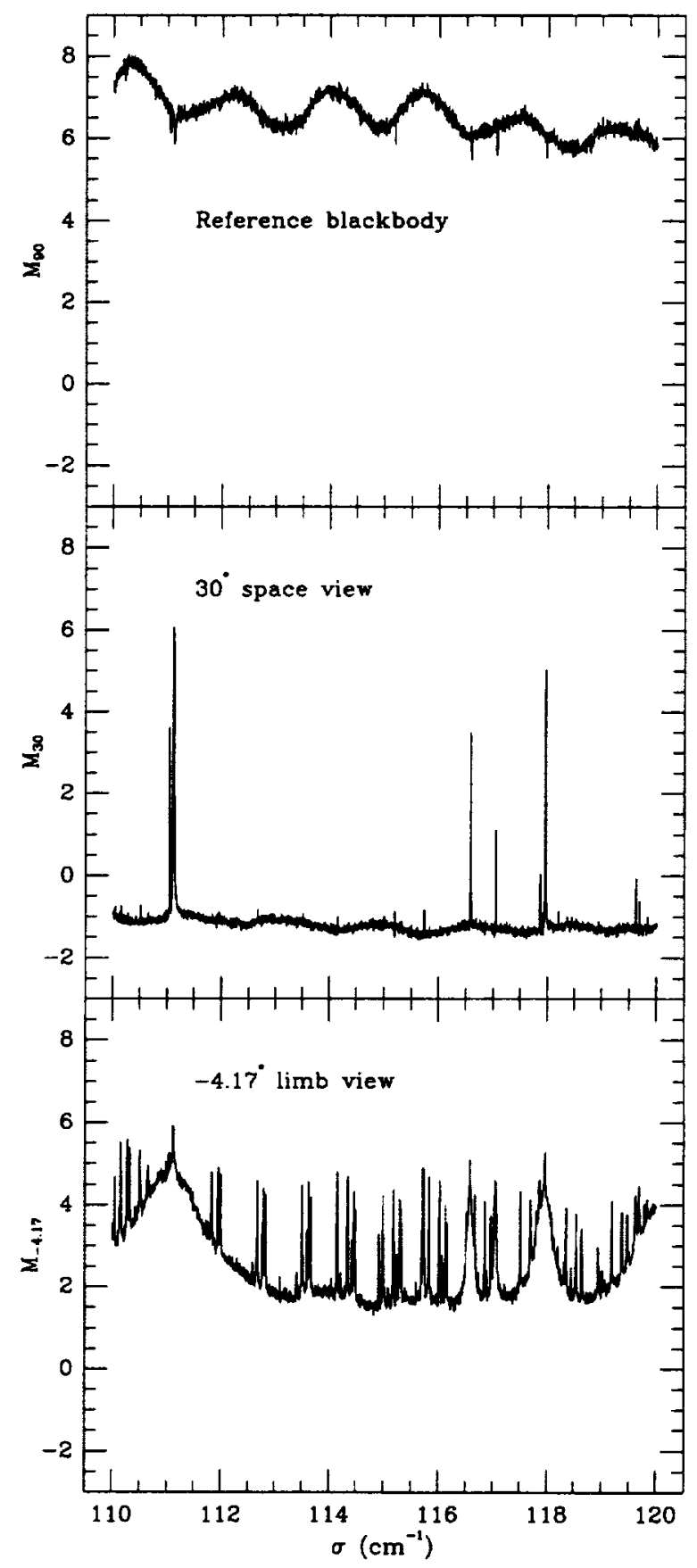

Fig. 1. FIRS-2 spectra recorded at a balloon altitude of $36 \mathrm{~km}$. Vertical scale (arbitrary units) is the same for each graph. The elevation angles are indicated; at $90^{\circ}$ the telescope views a reference blackbody. 


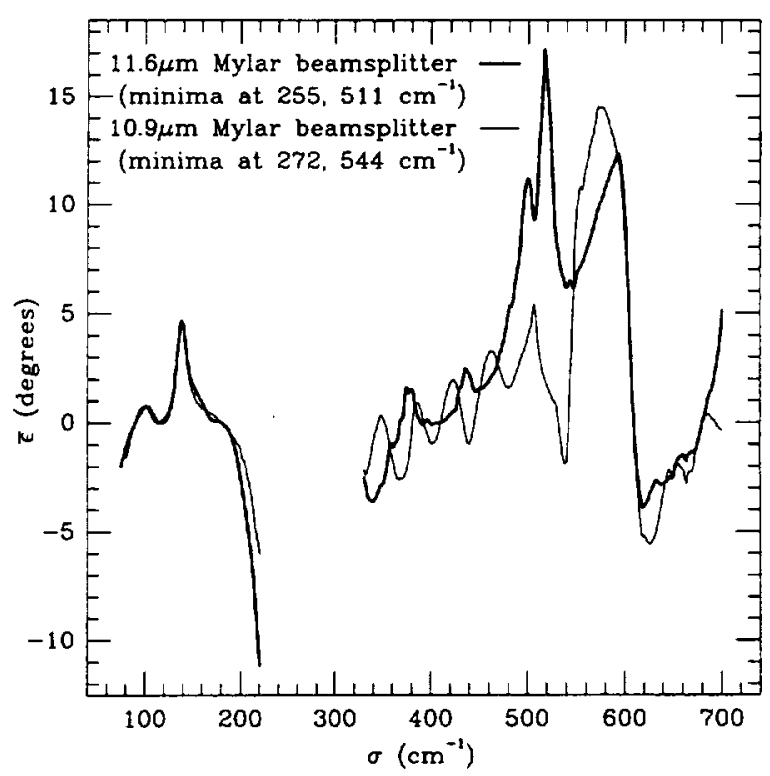

Fig. 2. Non-linear phase term $\bar{\epsilon}$ estimated for two different Mylar beamsplitters. The data from 75-220 are from the far-infrared detector, and data from 330-700 are from the mid-infrared detector.

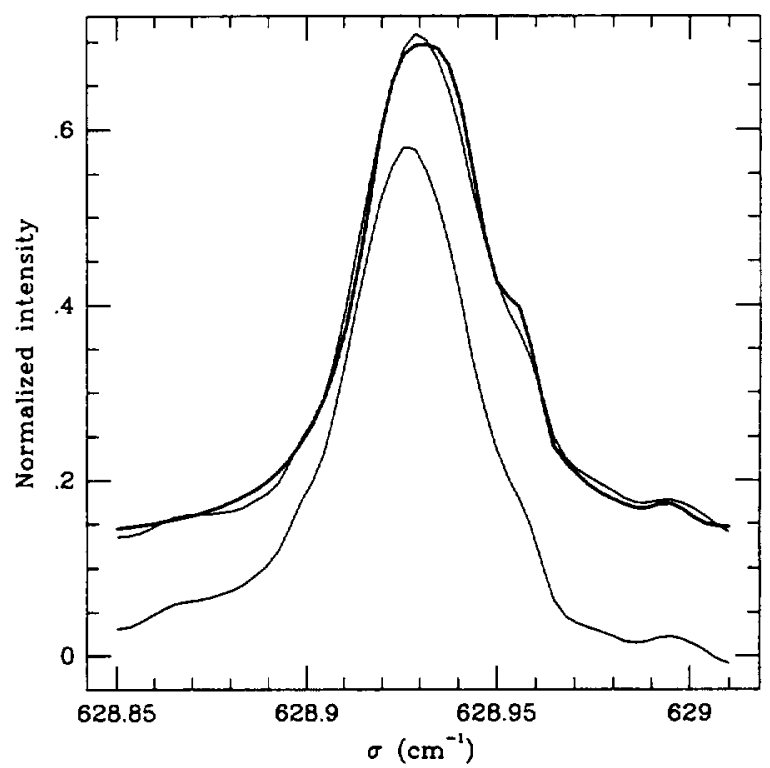

Fig. 3. A portion of a flight spectrum which shows the effect of ignoring the non-linear phase term $\bar{\epsilon}$. The upper curve was produced using the phase-recovery method outlined in this paper; the heavy curve indicates a calculated spectrum for the same region. The lower curve shows the result after assuming that $\bar{\epsilon}=0$. The baselines for the upper curve and calculated spectrum have been shifted by 0.12 for clarity. 


\section{APPENDIX B}

Ozone Production and Loss Rate Measurements in the Middle Stratosphere 